\title{
Yellowish discoloration of teeth and nails during oral iron therapy in a pregnant woman
}

\author{
R. Pai, ${ }^{1}$ S. Palaian, ${ }^{2}$ K. Alam, ${ }^{2}$ S. Prabhu, ${ }^{4}$ M. Prabhu, ${ }^{5}$ P. Mishra ${ }^{3}$ \\ ${ }^{1}$ Department of Orthopedics, ${ }^{2}$ Department of Pharmacology, ${ }^{3}$ Department of Hospital and Clinical Pharmacy, \\ Manipal Teaching Hospital / Manipal College of Medical Sciences Pokhara, Nepal, \\ ${ }^{4}$ Department of Dermatology, ${ }^{5}$ Department of Medicine, Kasturba Medical College Manipal, Karnataka, India.
}

Correspondence to: Rajaram Pai, Department of Orthopedics, Manipal Teaching Hospital / Manipal College of Medical Sciences, Pokhara, Nepal. Phone: +977 61526416 Extn: 221

Email: dic.mth@manipal.edu.np

\begin{abstract}
A 27 year old pregnant woman was prescribed conventional oral iron tablets from second trimester of pregnancy. She developed gastrointestinal symptoms within 1-2 days. Following these symptoms, Iron tablets were stopped and the patient was started with iron capsule. The patient tolerated this preparation well and after 6 months she developed yellowish discoloration of the teeth and nails. Iron treatment was suspected for the discolorations and the drug was stopped. Upon stopping the therapy the discoloration vanished and teeth and nails returned to the pre treatment condition. The causality assessment and the severity assessments were carried out as per the Naranjo and Hartwig scales respectively. Since Iron treatment during pregnancy is common, this adverse drug reaction warrants monitoring.
\end{abstract}

Keywords: Iron, nail discoloration, pregnancy, teeth discoloration

\section{Introduction}

Iron exists largely in the environment as ferrous oxide or hydroxide or as polymers. Oral iron preparations are used to treat conditions associated with iron deficiency like anemia and in pregnancy. ${ }^{1}$ Common side effects due to oral iron preparations are gastric irritation, nausea, epigastric pain, altered bowel habit. ${ }^{2}$ Clinical use of liquid iron products are known to cause extrinsic staining of teeth while given in the form of antiseptic or caries-preventing mouth rinses or as nutritional supplements. ${ }^{3}$ Discoloration of nails due to drugs is rarely reported in the literature. We here by report a case of yellowish discoloration of teeth and nails in a pregnant woman caused due to oral iron preparation given in the form of capsules. We established the causality and severity of the Adverse Drug Reaction (ADR) as per the
Naranjo algorithm and Hartwig scales respectively. ${ }^{4,5}$

\section{Case report}

A 27 year old pregnant woman was given conventional iron preparation in the form of tablet during her second trimester of pregnancy. The patient developed gastrointestinal symptoms within 1-2 days, following which the tablet was substituted with a capsule form, which she tolerated well. The patient was fine till she developed yellowish discoloration of the teeth and nail after 6 months of therapy with oral iron (Fig.1). During that period, the lady was not on any other medications. Iron treatment was stopped and the discoloration completely subsided within a period of 2 months (Fig. 2). The causality assessment of the adverse drug reaction was carried out and was found to be probable 
(score 6) as per the Naranjo algorithm and the severity to be mild (level 2) as per the Hartwig scale. ${ }^{4,5}$

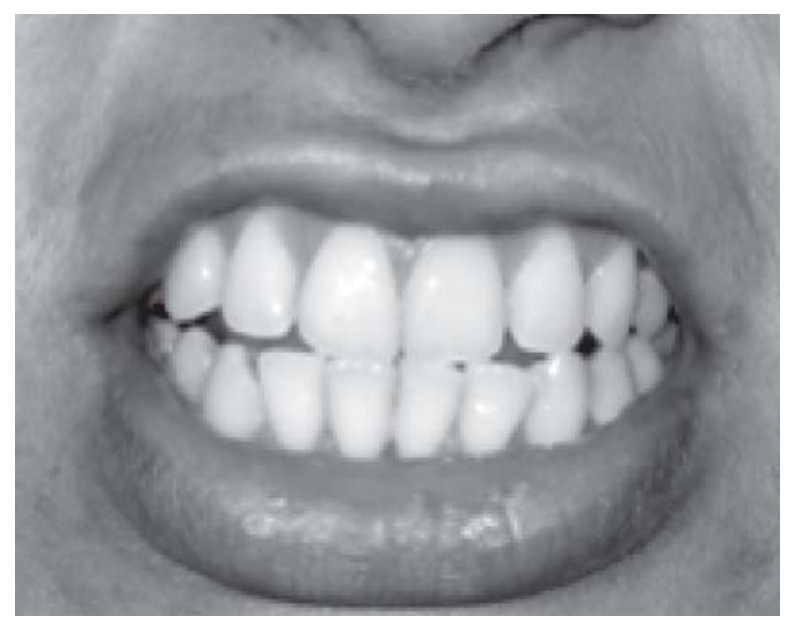

Fig. 1: Yellowish discoloration of the teeth during therapy

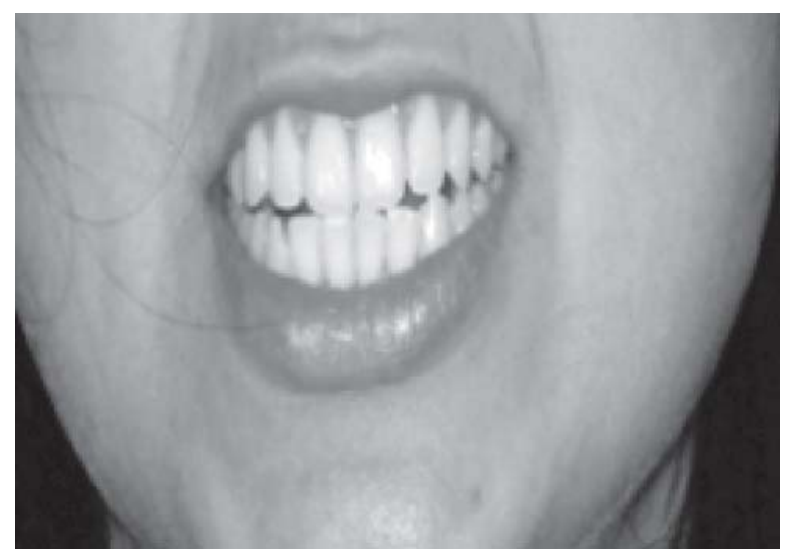

Fig. 2: The discoloration in the teeth disappeared after stopping of the drug

\section{Discussion}

The daily iron requirement for men is approximately $1 \mathrm{mg}$ whereas in menstruating woman is nearly $1.4 \mathrm{mg} .{ }^{6}$ This requirement of iron is fulfilled by the normal diet. However supplemental iron is used in infants, young children, adolescent females, pregnant women and especially in various iron deficiency disorders. Pregnant women have a two fold risk of anemia, which is in turn associated with premature delivery and three fold risk of low birth weight babies. Although supplemental iron decreases the incidence of anemia, evidence on the adverse maternal and infant outcome is inconclusive. ${ }^{7}$ The United States Food and Drug Administration has categorized iron in pregnancy as a category B drug suggesting that animal studies do not indicate a risk to the fetus; however, there are no adequate, well-controlled studies in pregnant women. Animal studies have shown an adverse effect on the fetus but adequate, well-controlled studies in pregnant women have failed to demonstrate a risk to the fetus. Despite the animal findings, the possibility of fetal harm appears remote, if used during pregnancy. $^{3}$

Discoloration of teeth has been associated with a large number of drugs. Extrinsic discoloration of teeth may occur due to drugs like chlorohexidine, essential oils and coamoxiclav. However, intrinsic teeth discoloration is associated with fluorides, tetracycline, minocycline and ciprofloxacin. ${ }^{8}$ Reversible staining of teeth due to liquid iron preparation is reported in the literature. ${ }^{9}$ However, we did not find conclusive report on staining of teeth due to systemic Iron through capsule preparation. The causes of extrinsic yellow staining of teeth are suggested as moderate trauma to teeth during enamel formation (secondary teeth) such as turner tooth, periapical infection of primary tooth, traumatic injury to primary tooth or teeth, trauma without hemorrhage, composites or glass ionomer or acrylic restoration, caries (active), focal tooth abrasion etc. ${ }^{10}$ There are frequent reports suggesting the yellowing of nail in different disease condition like rheumatoid arthritis, psoriasis and AIDS. ${ }^{11,12}$ Yellowing of nail is found during therapy with drugs like D-pencillinamine and gold salt. ${ }^{13,14}$ We could not locate any reports regarding nail discoloration due to iron therapy. In our patient, the ADR developed after starting of the drug and disappeared after the withdrawal of drug. Hence the diagnosis of yellowish discoloration of teeth and nails due to oral Iron capsule is a likely possibility.

The causal relation between the drugs and ADR was established and was found to be possible as per the Naranjo scale and severity of the reaction was found as mild (Level 2) suggesting that the ADR requires the suspected drug be withheld, discontinued, or otherwise changed. No antidote or other treatment is required, and there is no increase in length of stay in the hospital.

\section{Conclusions}

Iron treatment during pregnancy is very important. Teeth and nails discoloration can be a complication of iron supplementation. We recommend that pregnant women prescribed with iron preparations should be monitored for this effect. 
R. Pai, S. Palaian, K. Alam, et. al.

\section{References}

1. Sweetman SC. editor. Martindale The Complete Drug Reference. 33rd ed. London: Pharmaceutical Press; 2002.

2. Mehta DK, Martin J, Costello I, Jordan B, et al, editors. BNF 50. London: BMJ publishing group; 2005

3. Klasco RK (Ed): DRUGDEX® System. Thomson Micromedex, Greenwood Village, Colorado (Edition expires [6/2006])

4. Naranjo CA, Busto U, Sellers EM, Sandor P, Ruiz I, Roberts EA, et al. A method for estimating the probability of adverse drug reactions. Clin Pharmacol Ther 1981;30:239-45.

5. Hartwig SC, Siegel J, Schneider PJ. Preventability and severity assessment in reporting adverse drug reactions. Am J Hosp Pharm 1992;49:2229-32.

6. Kaushansky K, Kipps TJ. Hematopoietic agents: Growth factors, minerals and vitamins. In: Brunton LL, Lazo JS, Parker KL. Goodman and Gilman's the pharmacological basis of therapeutics. 11th ed. New York: McGraw-Hill; 2006. p. 1433-65.

7. McEvoy GK, Miller J, Litvak K, et al, editors. AHFS Drug Information. United States of America: American Society of Health-System Pharmacists; 2003.

8. Tredwin CJ, Scully C, Bagan-Sebastian JV. Druginduced disorders of teeth. J Dent Res 2005;84(7): 596-602.

9. Dukes MNG, Aronson JK, editors Meyler's side effects of drugs. 14th ed. Amsterdam: Elsevier; 2000.

10. Ship JA, Kerr AR. Tooth discoloration. [online]. eMedicine. [Cited 2006 Oct 8]. Available from: URL:http:/www.emedicine.com/derm/topic646.htm

11. Lehuede G, Toussirot E, Despaux J, Michel F, Wendling D. Yellow nail syndrome associated with thiol compound therapy for rheumatoid arthritis. Two case reports. Joint Bone Spine 2002;69(4):406-8.

12. Chernosky ME, Finley VK. Yellow nail syndrome in patients with acquired immunodeficiency disease. J Am Acad Dermatol 1985; 13 (5 Pt 1): 731-6.

13. Lubach D, Marghescu S. Yellow-nail syndrome from d-penicillamine. Hautarzt 1979; 30(10):547-9.

14. Roest MA, Ratnavel R. Yellow nails associated with gold therapy for rheumatoid arthritis. Br J Dermatol 2001;145(5):855-6. 